\title{
On fluctuations and localization length for the Anderson model on a strip
}

\author{
Ilia Binder, Michael Goldstein, and Mircea Voda
}

\begin{abstract}
We consider the Anderson model on a strip. Assuming that potentials have bounded density with considerable tails we get a lower bound for the fluctuations of the logarithm of the Green's function in a finite box. This implies an effective estimate by $\exp \left(C W^{2}\right)$ for the localization length of the Anderson model on the strip of width $W$. The results are obtained, actually, for a more general model with a non-local operator in the vertical direction.
\end{abstract}

Mathematics Subject Classification (2010). Primary 82B44; Secondary 47B36, 81Q10.

Keywords. Random Schrödinger operators, Anderson localization, multiscale analysis, fluctuations of Green's function.

\section{Contents}

1 Introduction . . . . . . . . . . . . . . . . . . . . 194

2 Lower bound for the variance of the logarithm of a rational function of several variables . . . . . . . . . . . . . . . . . . . 198

3 Analysis of the determinant and of the minors as polynomials in terms of the potentials . . . . . . . . . . . . . . . 209

4 Large Fluctuations Imply Exponential Decay . . . . . . . . . . . . . . 214

A Cartan's Estimate . . . . . . . . . . . . . . 221

B Resolvent Identities . . . . . . . . . . . . . . . . 223

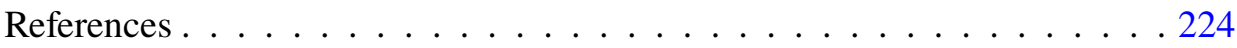




\section{Introduction}

We consider random operators on the strip $\mathbb{Z}_{W}=\mathbb{Z} \times\{1, \ldots, W\}$ defined by

$$
(H \psi)_{n}=-\psi_{n-1}-\psi_{n+1}+S_{n} \psi_{n},
$$

where

$$
\psi \in l^{2}\left(\mathbb{Z}, \mathbb{C}^{W}\right) \equiv l^{2}\left(\mathbb{Z}_{W}\right),
$$

and

$$
S_{n}=S+\operatorname{diag}\left(V_{(n, 1)}, \ldots, V_{(n, W)}\right),
$$

with $S$ a Hermitian matrix and $V_{i}, i \in \mathbb{Z}_{W}$, i.i.d. random variables. We assume that $V_{i}$ have bounded density function $v$ and we let

$$
A_{0}:=\sup _{x} v(x)<+\infty .
$$

Furthermore we assume that

$$
\mathbb{P}\left(\left|V_{i}\right| \geq T\right) \leq A_{1} / T,
$$

for $T \geq 1$.

The problem of estimating the localization length for this model and for the random band matrix model is well-known. In the latter case a polynomial bound was established by Schenker [7]. Very recently, Bourgain [2] established a bound by $\exp \left(C W(\log W)^{4}\right)$ for the Anderson model, provided that the potentials $V_{i}$ have bounded density. We will obtain an explicit estimate for the localization length by a method different from [2]. Our approach is via explicit lower bounds for the fluctuations of the Green's function. This idea has been previously used by Schenker [7], but our implementation is different.

We introduce some notation needed to state our results. Let $\Lambda \subset \mathbb{Z}_{W}$. For $\Lambda_{0} \subset \Lambda$ we let $\Lambda_{0}^{\prime}=\Lambda \backslash \Lambda_{0}$ and we use $\partial_{\Lambda} \Lambda_{0}$ to denote the boundary of $\Lambda_{0}$ relative to $\Lambda$, which is the set of pairs $\left(i, i^{\prime}\right)$ such that $i \in \Lambda_{0}, i^{\prime} \in \Lambda_{0}^{\prime}$, and $\left|i-i^{\prime}\right|=1$, where $|j|=\max \left(\left|j_{1}\right|,\left|j_{2}\right|\right)$. If $\Lambda=\mathbb{Z}_{W}$ we will just write $\partial \Lambda_{0}$. If $\left(i, i^{\prime}\right) \in \partial_{\Lambda} \Lambda_{0}$ we may also write $i \in \partial_{\Lambda} \Lambda_{0}$ and $i^{\prime} \in \partial_{\Lambda} \Lambda_{0}$. By $P_{\Lambda}$ we denote the orthogonal projection onto the subspace of all vectors in $\mathbb{C}^{\Lambda}$ vanishing off $\Lambda$. The restriction of $H$ to $\Lambda$ with Dirichlet boundary conditions is the operator

$$
H_{\Lambda}: \mathbb{C}^{\Lambda} \longrightarrow \mathbb{C}^{\Lambda},
$$

defined by

$$
H_{\Lambda}:=P_{\Lambda} H P_{\Lambda} .
$$


For $E \subset \mathbb{Z}$ we use $E_{W}$ do denote $E \times\{1, \ldots, W\}$. We will use $\Lambda_{L}(a)$ to denote $[a-L, a+L]_{W}$. Finally, let

$$
\Sigma_{\Lambda}^{E}:=\sum_{i, j \in \partial \Lambda, i_{1}<j_{1}}\left|G_{\Lambda}^{E}(i, j)\right|^{2},
$$

where

$$
G_{\Lambda}^{E}=\left(H_{\Lambda}-E\right)^{-1}
$$

Note that for $\Lambda=[a, b]_{W}$ the above sum is over $i \in\{a\}_{W}$ and $j \in\{b\}_{W}$.

Our estimate on the fluctuations of the resolvent, which will be proved in Section 3 , is as follows.

Theorem 1.1. There exist constants $C_{0}, C_{1}=C_{1}\left(A_{0},|E|,\|S\|\right)$ such that for any $\Lambda=[a, b]_{W}$ we have

$$
\operatorname{Var}\left(\log \Sigma_{\Lambda}^{E}\right) \geq(b-a-1)\left(\inf _{I} v\right)^{W},
$$

where $I=\left[ \pm \exp (C K), \pm \exp \left(\left(C+C_{0}\right) K\right)\right]$, with $C \geq C_{1}$.

The above estimate would work with $G_{\Lambda}^{E}(i, j), i \in\{a\}_{W}, j \in\{b\}_{W}$, instead of $\Sigma_{\Lambda}^{E}$, but we need the result as is to be able to deduce exponential decay. Indeed, employing standard multi-scale analysis, as in [9], we show in Theorem 4.4 that if $\operatorname{Var}\left(\Sigma_{\Lambda}^{E}\right) \geq(b-a+1) \delta_{0}, \delta_{0}=\delta_{0}(W)$, then the localization length is roughly $\delta_{0}^{-C}$. Thus, in principle, estimating the fluctuations of $\Sigma_{\Lambda}^{E}$ can lead to polynomial bounds on the localization length. In this paper we only manage to obtain exponential bounds on the localization length. Concretely, Theorem 1.1 and Theorem 4.4 imply the following estimate on the off-diagonal decay of Green's function.

Theorem 1.2. Fix $B>0$ and $\beta \geq 1$. There exists a constant

$$
C_{0}=C_{0}\left(A_{0}, A_{1}, B, \beta,|E|,\|S\|\right)
$$

such that if $\inf _{I} v \geq \exp (-B W)$ for some $I$ as in Theorem 1.1 then

$$
\mathbb{P}\left(\log \left|G_{\Lambda_{L}(a)}^{E}(i, j)\right| \leq-\exp \left(-C_{0} W^{2}\right) L, \quad i \in\{a\}_{W}, j \in \partial \Lambda_{L}(a)\right) \geq 1-L^{-\beta},
$$

for any $L \geq \exp \left(2 C_{0} W^{2}\right)$ and $a \in \mathbb{Z}$. 
Remark. It is well known, and otherwise straightforward to deduce, that the above estimate implies exponential decay of the extended eigenvectors of $H$, and a lower bound on the non-negative Lyapunov exponents. Namely, we have that if $\gamma_{W}^{E}$ is the lowest non-negative Lyapunov exponent then $\gamma_{W}^{E} \geq \exp \left(-C W^{2}\right)$, and if $\psi$ is an extended eigenvector of $H$ then

$$
\limsup _{|i| \rightarrow \infty}(\log |\psi(i)|) /|i| \leq-\exp \left(-C W^{2}\right) .
$$

Let us discuss some of the ideas behind the proof of Theorem 1.1. The strategy is to take advantage of the fact that $G_{\Lambda}^{E}(i, j)$ is the ratio of two polynomials of different degrees in $\left(V_{i}\right)_{i \in \Lambda}$. We illustrate this idea in a simpler setting. If $P(x), Q(x)$ are two monic polynomials of one variable then $\log |P(x) / Q(x)| \simeq$ (deg $P-\operatorname{deg} Q$ ) $\log |x|$, provided $|x|$ is large enough. If $\operatorname{deg} P \neq \operatorname{deg} Q$ and large values of $|x|$ are taken with non-zero probability then the previous remark should be enough to capture some of the fluctuations of $\log |P(x) / Q(x)|$.

The above idea is not sufficient to generate the crucial factor $(b-a-1)$ in the lower bound on variance. Let $\left\{\Lambda_{k}\right\}$ be a partition of $\Lambda$ and let

$$
h_{k}(V)=\mathbb{E}\left(\log \left|G_{\Lambda}^{E}(i, j)(V, \cdot)\right|\right), \quad V \in \mathbb{R}^{\Lambda_{k}}
$$

(we keep the potentials on $\Lambda_{k}$ fixed and we average the rest). Then we have the following Bessel type inequality (see Lemma 2.1 (ii)):

$$
\operatorname{Var}\left(\log \left|G_{\Lambda}^{E}(i, j)\right|\right) \geq \sum_{k} \operatorname{Var}\left(h_{k}\right) .
$$

So, the problem is reduced to estimating the fluctuations of $h_{k}$. We obtain the factor $(b-a-1)$ by just choosing a fine enough partition. Ideally we would choose $\Lambda_{k}=\{k\}$, but this turns out to be incompatible with our first idea. Using hyper-spherical coordinates we can write

$G_{\Lambda}^{E}(i, j)\left(V, V^{\prime}\right)=G_{\Lambda}^{E}(i, j)\left(r, \xi, V^{\prime}\right), \quad V \in \mathbb{R}^{\Lambda_{k}}, V^{\prime} \in \mathbb{R}^{\Lambda_{k}^{\prime}}, r \in \mathbb{R}, \xi \in S^{\left|\Lambda_{k}\right|-1}$.

Let $d_{1}, d_{2}$ be the degrees of the numerator and denominator of $G_{\Lambda}^{E}\left(r, \xi, V^{\prime}\right)$ as polynomials in $r$. It is then not hard to see that the problem of finding a lower bound for $\operatorname{Var}\left(h_{k}\right)$ can be reduced to the problem of estimating the variance of a function of the form

$$
d_{1} \int_{\mathbb{C}} \log |r-\zeta| d \mu_{1}(\zeta)-d_{2} \int_{\mathbb{C}} \log |r-\zeta| d \mu_{2}(\zeta),
$$


where $\mu_{1}, \mu_{2}$ are probability measures. Note that if $\mu_{i}(|\zeta| \geq R)=0, i=1,2$, then the above function is approximately $\left(d_{1}-d_{2}\right) \log r$, for $r \gg R$, which leads us back to our first idea. Clearly, we want $d_{1} \neq d_{2}$. This is false for $\Lambda_{k}=\{k\}$, $k \in \Lambda$, but it turns out to be true for $\Lambda_{k}=\{k\}_{W}, k \in(a, b)$. The conditions $\mu_{i}(|\zeta| \geq R)=0, i=1,2$, turn out be roughly equivalent to the polynomials on the top and bottom of $G_{\Lambda}^{E}(i, j)\left(V, V^{\prime}\right)$ not vanishing for $V$ outside the ball of radius $R$ in $\mathbb{C}^{\Lambda_{k}}$ and all $V^{\prime} \in \mathbb{R}^{\Lambda_{k}^{\prime}}$. Unfortunately we can establish such a property only for the denominator of $G_{\Lambda}^{E}(i, j)$ (see Proposition 3.2). This is because the denominator is the determinant of a self-adjoint matrix, but the numerator is the determinant of a non-self-adjoint matrix. We circumvent this problem at the cost of a worse lower bound on variance. At a technical level this is a accounted for by the difference between statements (iii) and (v) of Proposition 2.2.

Finally, the ideas discussed above are synthesized in the following theorem, which will be proved in Section 2. If $P$ is a polynomial of $N$ variables and $J \subset$ $\{1, \ldots, N\}$ then $\operatorname{deg}_{J} P$ denotes the cumulative degree of $P$ with respect to the variables indexed by $J$. We will use $J^{\prime}$ to denote $\{1, \ldots, N\} \backslash J$. By $\left(x, x^{\prime}\right)$, $x \in \mathbb{R}^{J}, x^{\prime} \in \mathbb{R}^{J^{\prime}}$, we denote the vector in $\mathbb{R}^{J \cup J^{\prime}}$ with the components indexed by $J$ given by $x$ and the components indexed by $J^{\prime}$ given by $x^{\prime}$.

Theorem 1.3. Let $P$ and $Q$ be two polynomials of $N$ variables. Assume that the following conditions hold:

(a) there exist $J_{k} \subset\{1, \ldots, N\}, k=1, \ldots, N^{\prime}, J_{k} \cap J_{k^{\prime}}=\emptyset$ for $k \neq k^{\prime}$, $\left|J_{k}\right|=K$ such that

$$
0 \leq \operatorname{deg}_{J_{k}} P<\operatorname{deg}_{J_{k}} Q=K
$$

(b) for each $k$ and each $T \gg 1$ there exists $\mathcal{B}(k, T) \subset \mathbb{R}^{J_{k}^{\prime}}$ with $\mathbb{P}(\mathcal{B}(k, T)) \leq$ $B_{0} K^{2} T^{-1}$, such that for any $x^{\prime} \in \mathbb{R}^{J_{k}^{\prime}} \backslash \mathcal{B}(k, T)$ and any $x \in \mathbb{C}^{J_{k}}$ with $\min _{i}\left|x_{i}\right| \geq T$ we have $Q\left(x, x^{\prime}\right) \neq 0$.

Then there exist $C_{0}, C_{1}=C_{1}(D)$ such that

$$
\operatorname{Var}(\log (|P| /|Q|)) \geq N^{\prime}\left(\inf _{I} v\right)^{K}
$$

for any $I=\left[ \pm \exp (C K), \pm \exp \left(\left(C+C_{0}\right) K\right)\right]$, with $C \geq C_{1}$.

Acknowledgments. The authors are grateful to the anonymous referee for his helpful comments. 


\section{Lower bound for the variance}

\section{of the logarithm of a rational function of several variables}

In this section we will prove Theorem 1.3. The main idea for the proof is to reduce the analysis of the variance to the case of a one dimensional logarithmic potential for which we have the estimates from Proposition 2.2. But first we collect some elementary facts concerning the variance. We leave the proofs as an exercise for the reader.

Lemma 2.1. Let $(\Omega, \mathcal{F}, \mu)$ be a probability space.

(i) If $X, Y$ are square summable random variables then

$$
\left|\operatorname{Var}^{1 / 2}(X)-\operatorname{Var}^{1 / 2}(Y)\right| \leq \operatorname{Var}^{1 / 2}(X \pm Y)
$$

and

$$
|\operatorname{Var}(X)-\operatorname{Var}(Y)| \leq \mathbb{E}\left((X-Y)^{2}\right)^{1 / 2}\left(\mathbb{E}\left(X^{2}\right)^{1 / 2}+\mathbb{E}\left(Y^{2}\right)^{1 / 2}\right) .
$$

(ii) If $X$ is a square summable random variable and $\mathcal{F}_{i}, i=1, \ldots, n$ are pairwise independent $\sigma$-subalgebras of $\mathcal{F}$ then

$$
\operatorname{Var}(X) \geq \sum_{i=1}^{n} \operatorname{Var}\left(\mathbb{E}\left(X \mid \mathcal{F}_{i}\right)\right) .
$$

(iii) If $X$ is a square summable random variable and $\mu_{0}$ is a probability measure such that $\mu \geq c \mu_{0}$, with $c \in(0,1)$, then

$$
\operatorname{Var}(X) \geq c \operatorname{Var}_{\mu_{0}}(X) .
$$

(iv) If $\mu_{i}, i=1, \ldots, n$ are probability measures and $X_{j}, j=1, \ldots, m$ are square summable random variables then

$$
\sum_{i} \operatorname{Var}_{\mu_{i}}\left(\sum_{j} \beta_{j} X_{j}\right) \leq\left(\sum_{j}\left|\beta_{j}\right|\right)^{2} \max _{j} \sum_{i} \operatorname{Var}_{\mu_{i}}\left(X_{j}\right) .
$$

(v) If $\left(\Omega^{\prime}, \mathcal{F}^{\prime}, \mu^{\prime}\right)$ is a probability space and $X$ is a square summable random variable on $\Omega \times \Omega^{\prime}$ then

$$
\operatorname{Var}_{\mu \times \mu^{\prime}}(X) \geq \underset{\omega^{\prime} \in \Omega^{\prime}}{\operatorname{essinf}} \operatorname{Var}_{\mu}\left(X\left(\cdot, \omega^{\prime}\right)\right) .
$$


From now on we will reserve $d v$ for the joint probability distribution of $\left(V_{i}\right)_{i \in \Lambda}$, where $\Lambda$ will be clear from the context. We use $d m_{\Omega}$ for the uniform distribution on $\Omega \subset \mathbb{R}^{d}$ (with $d$ clear from the context) and $\operatorname{Var}_{\Omega}(\cdot), \mathbb{E}_{\Omega}(\cdot)$ will be computed with respect to $d m_{\Omega}$. The statement of the next result exposes the main steps of its proof. We note that the statements relevant for the proof of Theorem 1.3 are (iii) and (v).

Proposition 2.2. Let $\mu$ be a Borel probability measure on $\mathbb{C}$ and set

$$
u_{\mu}(x):=\int_{\mathbb{C}} \log |x-\zeta| d \mu(\zeta)
$$

We assume that $\mu$ is such that $u_{\mu}$ is locally square summable.

(i) If $\mu(\{|\zeta| \geq R\})=0$ for some $R>0$, then, for any $M>0$,

$$
\mathbb{E}_{[0, M]}\left(u_{\mu}^{2}\right) \leq \frac{4 \min (1, M)(\log (\min (1, M))-1)^{2}+M \log ^{2}(M+R)}{M} .
$$

(ii) For any $M_{1}>M_{0} \geq 0$,

$$
\operatorname{Var}_{\left[M_{0}, M_{1}\right]}\left(u_{\mu}\right)=\operatorname{Var}_{\left[M_{0} M_{1}^{-1}, 1\right]}\left(u_{\left.\mu^{\left(M_{1}\right)}\right)},\right.
$$

where $\mu^{\left(M_{1}\right)}(\cdot):=\mu\left(M_{1} \cdot\right)$.

(iii) If $\mu(\{|\zeta| \geq R\})=0$ for some $R>0$, then for, any $M_{1} \geq 2 M_{0} \geq 4 R$,

$$
\left|\operatorname{Var}_{\left[M_{0}, M_{1}\right]}\left(u_{\mu}\right)-1\right| \leq 10^{4}\left(\left(R M_{1}^{-1}\right)^{1 / 5}+\left(M_{0} M_{1}^{-1}\right)^{1 / 2}\right) .
$$

(iv) If $\mu(\{|\zeta| \leq R\})=0$ for some $R>0$, then, for any $0 \leq 2 M_{0} \leq M_{1} \leq R / 2$,

$$
\operatorname{Var}_{\left[M_{0}, M_{1}\right]}\left(u_{\mu}\right) \leq 8\left(M_{1} R^{-1}\right)^{2} .
$$

(v) For any $M_{0} \geq 0$,

$$
\sum_{k=1}^{m} \operatorname{Var}_{\left[M_{0}, M_{k}\right]}\left(u_{\mu}\right)<m+10^{5},
$$

with $M_{k}=2^{k} A_{0}, A_{0}>0, A_{0} \geq M_{0}$. In particular, for any $m \geq 1$, there exists $M \in\left[2 A_{0}, 2^{m} A_{0}\right]$ such that $\operatorname{Var}_{\left[M_{0}, M\right]}\left(u_{\mu}\right)<1+10^{5} m^{-1}$. 
Proof. Recall that, for $A>0$,

$$
\int_{0}^{A} \log x d x=A(\log A-1)
$$

and

$$
\int_{0}^{A} \log ^{2} x d x=A\left[(\log A-1)^{2}+1\right] .
$$

(i) We have

$$
\begin{aligned}
\mathbb{E}_{[0, M]}\left(u_{\mu}^{2}\right) \leq & \frac{1}{M} \int_{0}^{M} \int_{|\zeta| \leq R}(\log |x-\zeta|)^{2} d \mu(\zeta) d x \\
= & \frac{1}{M} \int_{|\zeta| \leq R}\left(\int_{x \in[0, M],|x-\zeta|<1}(\log |x-\zeta|)^{2} d x\right. \\
& \left.\quad+\int_{x \in[0, M],|x-\zeta| \geq 1}(\log |x-\zeta|)^{2} d x\right) d \mu(\zeta) \\
\leq & \frac{1}{M} \int_{|\zeta| \leq R}\left(2 \int_{0}^{\min (1, M)}(\log y)^{2} d y+M(\log (M+R))^{2}\right) d \mu(\zeta) \\
\leq & \frac{4 \min (1, M)(\log (\min (1, M))-1)^{2}+M \log ^{2}(M+R)}{M}
\end{aligned}
$$

(ii) By a change of variables we have

$$
\operatorname{Var}_{\left[M_{0}, M_{1}\right]}\left(u_{\mu}\right)=\operatorname{Var}_{\left[M_{0} M_{1}^{-1}, 1\right]}\left(u_{\mu}\left(M_{1} \cdot\right)\right) .
$$

Now the conclusion follows from the fact that

$$
u_{\mu}\left(M_{1} x\right)=u_{\mu^{\left(M_{1}\right)}}(x)+\log M_{1} .
$$

(iii) First note that

$$
|\log | x-\zeta|-\log | x|| \leq 2|x|^{-1}|\zeta|,|x|^{-1}|\zeta| \leq 1 / 2,
$$

and consequently

$$
\left|u_{\mu^{\left(M_{1}\right)}}(x)-\log x\right| \leq 2 \sqrt{R M_{1}^{-1}}, \quad x \in\left[\sqrt{R M_{1}^{-1}}, 1\right] .
$$


By what we already established,

$$
\begin{aligned}
& \left|\operatorname{Var}_{\left[M_{0}, M_{1}\right]}\left(u_{\mu}\right)-\operatorname{Var}_{\left[M_{0}, M_{1}\right]}(\log )\right| \\
& \quad=\left|\operatorname{Var}_{\left[M_{0} M_{1}^{-1}, 1\right]}\left(u_{\mu^{\left(M_{1}\right)}}\right)-\operatorname{Var}_{\left[M_{0} M_{1}^{-1}, 1\right]}(\log )\right| \\
& \quad \leq\left\|u_{\mu^{\left(M_{1}\right)}}-\log \right\|_{L_{\left[M_{0} M_{1}^{-1}, 1\right]}^{2}\left(\left\|u_{\mu^{\left(M_{1}\right)}}\right\|_{L_{\left[M_{0} M_{1}^{-1}, 1\right]}^{2}}+\|\log \|_{L_{\left[M_{0} M_{1}^{-1}, 1\right]}^{2}}\right)} \\
& \quad \leq 2\left\|u_{\mu^{\left(M_{1}\right)}}-\log \right\|_{L_{[0,1]}^{2}} \underbrace{\left.\left\|u_{\mu^{\left(M_{1}\right)}}\right\|_{L_{[0,1]}^{2}}+\|\log \|_{L_{[0,1]}^{2}}\right)}_{<5} \\
& \quad \leq 10\left(4 R M_{1}^{-1}+\int_{0}^{\sqrt{R M_{1}^{-1}}} 2\left(u_{\mu^{\left(M_{1}\right)}}^{2}(x)+\log ^{2} x\right) d x\right)^{1 / 2} \\
& \quad \leq 10\left(4 R M_{1}^{-1}+350 \sqrt{R M_{1}^{-1}} \log ^{2} \sqrt{R M_{1}^{-1}}\right)^{1 / 2} \\
& \quad \leq 100\left(R M_{1}^{-1}\right)^{1 / 4} \log \left(M_{1} R^{-1}\right) \\
& \quad \leq 2000\left(R M_{1}^{-1}\right)^{1 / 5} .
\end{aligned}
$$

Now we just have to estimate

$$
\operatorname{Var}_{\left[M_{0}, M_{1}\right]}(\log )=\operatorname{Var}_{[m, 1]}(\log ),
$$

where we let $m=M_{0} M_{1}^{-1}$ :

$$
\begin{aligned}
& \left|\operatorname{Var}_{[m, 1]}(\log )-1\right| \\
& =\mid \mathbb{E}_{[m, 1]}\left(\log ^{2}\right)-\frac{1}{1-m} \mathbb{E}_{[0,1]}\left(\log ^{2}\right) \\
& -\left(\mathbb{E}_{[m, 1]}(\log )\right)^{2}+\frac{1}{(1-m)^{2}}\left(\mathbb{E}_{[0,1]}(\log )\right)^{2}-\frac{m^{2}}{(1-m)^{2}} \\
& \leq \frac{1}{1-m}\left|\int_{0}^{m}(\log x)^{2} d x\right| \\
& +\frac{1}{(1-m)^{2}}\left|\int_{0}^{m} \log x d x\right|\left|\int_{0}^{1} \log x d x+\int_{m}^{1} \log x d x\right| \\
& +\frac{m^{2}}{(1-m)^{2}} \\
& \leq \frac{5 m(1-\log m)^{2}}{(1-m)^{2}} \\
& \leq 500 m \log ^{2} m \\
& \leq 10^{4} m^{1 / 2} \text {. }
\end{aligned}
$$


(iv) Note that, based on (2.7),

$$
\left|u_{\mu^{\left(M_{1}\right)}}(x)-u_{\mu^{\left(M_{1}\right)}}(0)\right| \leq 2 M_{1} R^{-1}, \quad x \in\left[M_{0} M_{1}^{-1}, 1\right],
$$

and hence

$$
\begin{aligned}
\operatorname{Var}_{\left[M_{0}, M_{1}\right]}\left(u_{\mu}\right) & =\operatorname{Var}_{\left[M_{0} M_{1}^{-1}, 1\right]}\left(u_{\mu^{\left(M_{1}\right)}}\right) \\
& \leq\left\|u_{\mu^{\left(M_{1}\right)}}-u_{\mu^{\left(M_{1}\right)}}(0)\right\|_{L_{\left[M_{0} M_{1}^{-1}, 1\right]}^{2}}^{2} \\
& \leq \frac{4\left(M_{1} R^{-1}\right)^{2}}{1-M_{0} M_{1}^{-1}} \\
& \leq 8\left(M_{1} R^{-1}\right)^{2} .
\end{aligned}
$$

(v) Let

$$
\begin{aligned}
& D_{l}=\left\{M_{l} \leq|\zeta|<M_{l+1}\right\}, \quad l=1, \ldots, m-1, \\
& D_{0}=\left\{|\zeta|<M_{1}\right\},
\end{aligned}
$$

and

$$
D_{m}=\left\{|\zeta| \geq M_{m}\right\}
$$

We have

$$
u_{\mu}=\sum_{l=0}^{m} \mu\left(D_{l}\right) u_{\mu_{D_{l}}}
$$

where

$$
\mu_{D}=\left.\mu(D)^{-1} \mu\right|_{D}
$$

(we set $\mu_{D}=0$ if $\mu(D)=0$ ). We will verify the estimate in (v) for each measure $\mu_{D_{l}}$. The estimate for $\mu$ will follow by (2.5). So, fix arbitrary $l \in\{0, \ldots, m\}$. One has due to part (iv) that

$$
\sum_{k=1}^{l-1} \operatorname{Var}_{\left[M_{0}, M_{k}\right]}\left(u_{\mu_{D_{l}}}\right) \leq \sum_{k=1}^{l-1} 8\left(M_{k} M_{l}^{-1}\right)^{2}=8 \sum_{k=1}^{l-1} 4^{k-l} \leq 8 .
$$

On the other hand due to part (iii) one has

$$
\begin{aligned}
\sum_{k=l+3}^{m} \operatorname{Var}_{\left[M_{0}, M_{k}\right]}\left(u_{\mu_{D_{l}}}\right) & \leq \sum_{k=l+3}^{m}\left[1+10^{4}\left(\left(M_{0} M_{k}^{-1}\right)^{1 / 2}+\left(M_{l+1} M_{k}^{-1}\right)^{1 / 5}\right)\right] \\
& \leq m+10^{4}\left(\sum_{k=1}^{\infty} 2^{-k / 2}+\sum_{k=1}^{\infty} 2^{-k / 5}\right) \\
& \leq m+5 \cdot 10^{4}
\end{aligned}
$$


Now we just have to evaluate the variance for $l \leq k \leq l+2$. For $l<m$ we use (i) to get

$$
\begin{aligned}
\sum_{k=l}^{l+2} \operatorname{Var}_{\left[M_{0}, M_{k}\right]}\left(u_{\mu_{D_{l}}}\right) & =\sum_{k=l}^{l+2} \operatorname{Var}_{\left[M_{0} M_{k}^{-1}, 1\right]}\left(u_{\mu_{D_{l}}}^{\left(M_{k}\right)}\right) \\
& \leq \sum_{k=l}^{l+2} \frac{1}{1-M_{0} M_{k}^{-1}}\left\|u_{\mu_{D_{l}}}^{\left(M_{k}\right)}\right\|_{L_{[0,1]}^{2}}^{2} \\
& \leq 2 \sum_{k=l}^{l+2}\left(4+\log ^{2}\left(1+M_{l+1} M_{k}^{-1}\right)\right) \\
& \leq 40 .
\end{aligned}
$$

When $l=m$ we just need to evaluate $\operatorname{Var}_{\left[M_{0}, M_{m}\right]}\left(u_{\mu_{D m}}\right)$. Let

$$
D_{m}^{1}=\left\{M_{m} \leq|\zeta|<2 M_{m}\right\}
$$

and

$$
D_{m}^{2}=\left\{|\zeta| \geq 2 M_{m}\right\}
$$

Using (2.5) (i) (for $u_{\mu_{D_{m}^{1}}}$, as above) and (iv) (for $u_{\mu_{D_{m}^{2}}}$ ), we get

$$
\begin{aligned}
\operatorname{Var}_{\left[M_{0}, M_{m}\right]}\left(u_{\mu_{D m}}\right) & \leq \max \left(\operatorname{Var}_{\left[M_{0}, M_{m}\right]}\left(u_{\mu_{D_{m}^{1}}}\right), \operatorname{Var}_{\left[M_{0}, M_{m}\right]}\left(u_{\mu_{D_{m}^{2}}}\right)\right) \\
& \leq \max \left(4+\log ^{2}\left(1+2 M_{m} / M_{m}\right), 8\left(M_{m} /\left(2 M_{m}\right)\right)^{2}\right) \\
& \leq 10 .
\end{aligned}
$$

This concludes the proof.

Before we proceed with the proof of Theorem 1.3 we need the two following auxiliary results.

Lemma 2.3. If $P(x)=\sum_{|\alpha| \leq D} a_{\alpha} x^{\alpha}$ is a polynomial of $N$ variables such that $\max _{|\alpha| \leq D}\left|a_{\alpha}\right|=1$, and $\Omega \subset\left\{x \in \mathbb{R}^{N}:\|x\| \leq R_{0}\right\}, R_{0} \geq e$, is such that $\operatorname{mes}(\Omega)>1$, then

$$
\mathbb{E}_{\Omega}\left(\log ^{2}|P|\right) \lesssim D^{2} N^{2} \log ^{2}(N+1) \log ^{4} R_{0}
$$


Proof. The polynomial $P$ has at most $(N+1)^{D}$ monomials, so for $R \geq e$ we have

$$
\sup _{\|z\| \leq R} \log |P(z)| \leq \log \left(R^{D}(N+1)^{D}\right) \lesssim D \log (N+1) \log R .
$$

Lemma A.2 implies that

$$
\begin{aligned}
& \operatorname{mes}\left\{x \in \mathbb{R}^{N}:\|x\| \leq R, \log |P(x)| \leq C H D \log (N+1) \log (20 R)\right\} \\
& \quad \leq C^{N} R^{N} \exp (-H),
\end{aligned}
$$

for $H \gg 1$. The conclusion follows from Lemma A.3.

Lemma 2.4. Let $\sigma$ be the spherical measure on the $(n-1)$-sphere $S^{n-1}$.

$$
\sigma\left(\left\{\xi \in S^{n-1}: \min _{i}\left|\xi_{i}\right| \geq \varepsilon\right\}\right) \geq n 2^{n}(1-\sqrt{n} \varepsilon)^{n} .
$$

Proof. Let $\Theta$ be the set whose measure we want to estimate and let

$$
\Omega=\left\{x \in \mathbb{R}^{n}: 1 \leq \min _{i}\left|x_{i}\right|, \max _{i}\left|x_{i}\right| \leq 1 /(\sqrt{n} \varepsilon)\right\} .
$$

Then we have

$$
\Omega \subset\{r \xi: \xi \in \Theta, r \in[1,1 /(\sqrt{n} \varepsilon)]\},
$$

and the conclusion follows from

$$
\begin{aligned}
2^{n}\left(\frac{1}{\sqrt{n} \varepsilon}-1\right)^{n} & =\operatorname{mes}(\Omega) \\
& \leq \int_{\Theta} \int_{1}^{1 /(\sqrt{n} \varepsilon)} r^{n-1} d r d \sigma(\xi) \\
& \leq \frac{1}{n}\left(\frac{1}{\sqrt{n} \varepsilon}\right)^{n} \sigma(\Theta) .
\end{aligned}
$$

Proof of Theorem 1.3. Set

$$
h(x):=\log (|P(x)| /|Q(x)|) .
$$

Due to (2.3) one has

$$
\operatorname{Var}(h) \geq \sum_{k} \operatorname{Var}\left(\mathbb{E}\left(h \mid \mathfrak{J}_{k}\right)\right)=\sum_{k} \operatorname{Var}\left(h_{k}\right),
$$

where $\mathcal{J}_{k}$ is the $\sigma$-algebra corresponding to fixing the components with indices in $J_{k}$, and $h_{k}(x)=\mathbb{E}(h(x, \cdot)), x \in \mathbb{R}^{J_{k}}$. 
To provide a lower bound for $\operatorname{Var}\left(h_{k}\right)$ we will pass to a uniform distribution and we will use hyper-spherical coordinates to pass to a one-dimensional problem. Let

$$
I=\left[M_{0} /(2 \sqrt{K}), M\right],
$$

with $M=2^{10^{6}} K M_{0}, M_{0}=2 \sqrt{K} T, T=B_{0} \exp (C K)$, and $C \gg 10^{6}$. We define

$$
\Theta=\left\{\xi \in S^{K-1}: \min _{i} \xi_{i} \geq \frac{1}{2 \sqrt{K}}\right\}
$$

and

$$
\Omega=\left\{x \in \mathbb{R}^{K}: x=r \xi, r \in\left[M_{0}, M\right], \xi \in \Theta\right\} .
$$

The peculiar choice of $\Theta$ is so that we will be able to use the assumptions on $Q$. Note that for $x \in \Omega$ we have $x_{i} \in I$. Furthermore, by Lemma 2.4 we have $\sigma(\Theta) \geq K 2^{-K}$ and consequently $\operatorname{mes}(\Omega) \geq 2^{-K}\left(M^{K}-M_{0}^{K}\right)$. By (2.4),

$$
\begin{aligned}
\operatorname{Var}\left(h_{k}\right) & \geq\left(\inf _{I} v\right)^{K} \operatorname{mes}(\Omega) \operatorname{Var}_{\Omega}\left(h_{k}\right) \\
& \geq\left(\inf _{I} v\right)^{K} 2^{-K}\left(M^{K}-M_{0}^{K}\right) \operatorname{Var}_{\Omega}\left(h_{k}\right) .
\end{aligned}
$$

Changing variables to hyper-spherical coordinates,

$$
\operatorname{Var}_{\Omega}\left(h_{k}\right)=\operatorname{Var}_{\eta}\left(h_{k}\right) \text {, }
$$

where

$$
d \eta:=\frac{K r^{K-1} d r}{M^{K}-M_{0}^{K}} \times \frac{d \sigma}{\sigma(\Theta)}
$$

is the probability measure on $\mathcal{R}=\left[M_{0}, M\right] \times \Theta$. Using (2.4) we can pass to the uniform distribution on $\mathcal{R}$ :

$$
\operatorname{Var}_{\Omega}\left(h_{k}\right) \geq \frac{K\left(M-M_{0}\right) M_{0}^{K-1}}{M^{K}-M_{0}^{K}} \operatorname{Var}_{\mathcal{R}}\left(h_{k}\right) .
$$

Finally, due to (2.6) we have

$$
\operatorname{Var}_{\mathcal{R}}\left(h_{k}\right) \geq \underset{\xi \in \Theta}{\operatorname{essinf}} \operatorname{Var}_{\left[M_{0}, M\right]}\left(h_{k}(\cdot, \xi)\right),
$$

where $h_{k}(r, \xi)=h_{k}(r \xi)$. In conclusion we have

$$
\operatorname{Var}\left(h_{k}\right) \geq K\left(M-M_{0}\right) M_{0}^{K-1} 2^{-K}\left(\inf _{I} v\right)^{K} \underset{\xi \in \Theta}{\operatorname{essinf}} \operatorname{Var}_{\left[M_{0}, M\right]}\left(h_{k}(\cdot, \xi)\right) .
$$


To be able to use the assumption on $Q$ we want to work with a truncated version of $h_{k}$ obtained by averaging only on

$$
\mathcal{G}_{k}:=\mathbb{R}^{J_{k}^{\prime}} \backslash \mathcal{B}(k, T),
$$

Passing from the variance of $h_{k}$ to the variance of the truncated function will depend on having an explicit bound on the second moment of $h_{k}$. The bound will follow using Lemma 2.3 after an appropriate normalization. We know $P$ and $Q$ are polynomials in $r$ and we can write

$$
P\left(r, \xi, x^{\prime}\right)=\sum_{i} a_{i}\left(\xi, x^{\prime}\right) r^{i},
$$

and

$$
Q(x)=\sum_{i} b_{i}\left(\xi, x^{\prime}\right) r^{i}
$$

Let $A\left(\xi, x^{\prime}\right)=\max _{i}\left|a_{i}\left(\xi, x^{\prime}\right)\right|, B\left(\xi, x^{\prime}\right)=\max _{i}\left|b_{i}\left(\xi, x^{\prime}\right)\right|$, and define

$$
\begin{aligned}
& \hat{P}\left(r, \xi, x^{\prime}\right)=P\left(r, \xi, x^{\prime}\right) / A\left(\xi, x^{\prime}\right), \\
& \widehat{Q}\left(r, \xi, x^{\prime}\right)=Q\left(r, \xi, x^{\prime}\right) / B\left(\xi, x^{\prime}\right),
\end{aligned}
$$

and

$$
\hat{h}=\log |\widehat{P} / \hat{Q}| .
$$

These functions are well-defined for $\sigma \times \nu$-almost all $\left(\xi, x^{\prime}\right)$. From now on we fix $\xi$ such that the functions are well-defined for $v$-almost all $x^{\prime}$. Of course, this means $\xi$ must be outside a set of measure 0 , but this doesn't affect the essential infimum in (2.9). Since $\mathbb{E}(|\log | A(\xi, \cdot)||), \mathbb{E}(|\log | B(\xi, \cdot)||)<\infty$ we have

$$
\operatorname{Var}_{\left[M_{0}, M\right]}\left(h_{k}(\cdot, \xi)\right)=\operatorname{Var}_{\left[M_{0}, M\right]}\left(\hat{h}_{k}(\cdot, \xi)\right) \text {, }
$$

where

$$
\hat{h}_{k}(r, \xi)=h_{k}(r, \xi)-\mathbb{E}(\log |A(\xi, \cdot)|)+\mathbb{E}(\log |B(\xi, \cdot)|) .
$$

Using Lemma 2.3 we obtain

$$
\begin{aligned}
\mathbb{E}_{\left[M_{0}, M\right]}\left(\hat{h}_{k}^{2}(\cdot, \xi)\right) & =\int_{\left[M_{0}, M\right]}\left(\int_{\mathbb{R}_{k}^{J_{k}^{\prime}}} \hat{h}\left(r, \xi, x^{\prime}\right) d v\left(x^{\prime}\right)\right)^{2} d m_{\left[M_{0}, M\right]}(r) \\
& \leq \int_{\mathbb{R}^{\prime}{ }_{k}^{\prime}}\left(\int_{\left[M_{0}, M\right]} \hat{h}^{2}\left(r, \xi, x^{\prime}\right) d m_{\left[M_{0}, M\right]}(r)\right) d v\left(x^{\prime}\right) \\
& \lesssim K^{2} \log ^{4} M .
\end{aligned}
$$


We now introduce the truncated version of $\hat{h}_{k}$ :

$$
\tilde{h}_{k}(r, \xi)=\int_{\mathcal{G}_{k}} \hat{h}\left(r, \xi, x^{\prime}\right) \frac{d \nu\left(x^{\prime}\right)}{\mathrm{P}\left(\mathcal{G}_{k}\right)} .
$$

By the same argument as for $\hat{h}_{k}(\cdot, \xi)$ we have

$$
\mathbb{E}_{\left[M_{0}, M\right]}\left(\tilde{h}_{k}^{2}(\cdot, \xi)\right) \lesssim K^{2} \log ^{4} M
$$

and

$$
\mathbb{E}_{\left[M_{0}, M\right]}\left(\left(\hat{h}_{k}(\cdot, \xi)-\mathbb{P}\left(\mathcal{G}_{k}\right) \tilde{h}_{k}(\cdot, \xi)\right)^{2}\right) \lesssim \mathbb{P}(\mathcal{B}(k, T)) K^{2} \log ^{4} M .
$$

We now get

$$
\begin{aligned}
\left|\operatorname{Var}_{\left[M_{0}, M\right]}\left(\hat{h}_{k}(\cdot, \xi)\right)-\operatorname{Var}_{\left[M_{0}, M\right]}\left(\mathbb{P}\left(\mathcal{G}_{k}\right) \tilde{h}_{k}(\cdot, \xi)\right)\right| \\
\leq \mathbb{E}_{\left[M_{0}, M\right]}\left(\left(\hat{h}_{k}(\cdot, \xi)-\mathbb{P}\left(\mathcal{G}_{k}\right) \tilde{h}_{k}(\cdot, \xi)\right)^{2}\right)^{1 / 2} \\
\quad \cdot\left(\mathbb{E}_{\left[M_{0}, M\right]}\left(\hat{h}_{k}^{2}(\cdot, \xi)\right)^{1 / 2}+\mathbb{E}_{\left[M_{0}, M\right]}\left(\tilde{h}_{k}^{2}(\cdot, \xi)\right)^{1 / 2}\right) \\
\lesssim \\
\quad \mathbb{P}(\mathcal{B}(k, T))^{1 / 2} K^{2} \log ^{4} M .
\end{aligned}
$$

We claim that $\operatorname{Var}_{\left[M_{0}, M\right]}\left(\tilde{h}_{k}(\cdot, \xi)\right) \geq 2^{-10^{6} K}$. Since we chose

$$
T=B_{0} \exp (C K), C \gg 10^{6}
$$

it follows that

$$
\begin{aligned}
\operatorname{Var}_{\left[M_{0}, M\right]}\left(h_{k}(\cdot, \xi)\right) & \geq \mathbb{P}\left(\mathcal{G}_{k}\right)^{2} \operatorname{Var}_{\left[M_{0}, M\right]}\left(\tilde{h}_{k}(\cdot, \xi)\right)-C \mathbb{P}(\mathcal{B}(k, T))^{1 / 2} K^{2} \log ^{4} M \\
& \geq \operatorname{Var}_{\left[M_{0}, M\right]}\left(\tilde{h}_{k}(\cdot, \xi)\right) / 2 \\
& \geq 2^{-10^{6} K} / 2 .
\end{aligned}
$$

From this, (2.9), and (2.8) it follows that

$$
\operatorname{Var}(h) \geq N^{\prime} K\left(M-M_{0}\right) M_{0}^{K-1} 2^{-(K+1)} 2^{-10^{6} K}\left(\inf _{I} v\right)^{K} .
$$

Note that by our choice of $M_{0}, M, T$ we have

$$
K\left(M-M_{0}\right) M_{0}^{K-1} 2^{-(K+1)} 2^{-10^{6} K}=\exp \left(C K^{2}\right) \geq 1,
$$

so the desired lower bound on variance follows. The case

$$
I=\left[-M_{1},-M_{0} /(2 \sqrt{K})\right]
$$

follows analogously. Note that in fact we obtained a better estimate than the one stated in the theorem. However, it can be seen that $\left(\inf _{I} v\right)^{K} \leq \exp \left(-C^{\prime} K^{2}\right)$ with $C^{\prime} \gg C$, so the estimate won't be substantially better than the stated one. 
Now we just have to show that $\operatorname{Var}_{\left[M_{0}, M\right]}\left(\tilde{h}_{k}(\cdot, \xi)\right) \geq 2^{-10^{6} K}$. Using (2.4) we get

$$
\operatorname{Var}_{\left[M_{0}, M\right]}\left(\tilde{h}_{k}(\cdot, \xi)\right) \geq \frac{M_{\xi}-M_{0}}{M-M_{0}} \operatorname{Var}_{\left[M_{0}, M_{\xi}\right]}\left(\tilde{h}_{k}(\cdot, \xi)\right),
$$

with $M_{\xi} \in\left(M_{0}, M\right)$ to be chosen later.

We provide a lower bound for $\operatorname{Var}_{\left[M_{0}, M_{\xi}\right]}\left(\tilde{h}_{k}(\cdot, \xi)\right)$ by applying Proposition 2.2. We first need to set-up $\tilde{h}_{k}$ as the difference of two logarithmic potentials. Without loss of generality we may assume that $\widehat{P}$ and $\hat{Q}$ are monic polynomials in $r$ (we can force them to be so, without changing the variance). Let $D_{k}$ be the degree in $r$ of $\widehat{P}\left(r, \xi_{0}, x^{\prime}\right)$. If $D_{k}=0$ then the term corresponding to $\widehat{P}$ won't contribute to the variance. So, we only deal with the case $D_{k} \geq 1$. It is well-known that there exist measurable functions $\zeta_{j}$ such that

$$
\widehat{P}\left(r, \xi, x^{\prime}\right)=\prod_{j=1}^{D_{k}}\left(r-\zeta_{j}\left(x^{\prime}\right)\right) .
$$

Let $\mu_{j}$ be the push-forward of the measure $\left(\left.v\right|_{\mathcal{G}_{k}}\right) / \mathbb{P}\left(\mathcal{G}_{k}\right)$ under the map

$$
x^{\prime} \longmapsto \zeta_{j}\left(x^{\prime}\right) \text {. }
$$

Let

$$
u_{k}(r)=\int_{\mathbb{C}} \log |r-\zeta| d \mu_{P}(\zeta)
$$

where $\mu_{P}$ is the probability measure defined by

$$
\mu_{P}=D_{k}^{-1} \sum_{j} \mu_{j}
$$

Analogously, we define

$$
v_{k}(r)=\int_{\mathbb{C}} \log |r-\zeta| d \mu_{Q}(\zeta)
$$

to be the logarithmic potential corresponding to $\widehat{Q}\left(r, \xi_{0}, x^{\prime}\right)$. Note that both $u_{k}$ and $v_{k}$ are square summable, and furthermore by the choice of $\mathcal{G}_{k}$ and $\Theta$ we have $\mu_{Q}(|\zeta| \geq 2 \sqrt{K} T)=0$ (this is equivalent to saying that $\hat{Q}\left(r, \xi, x^{\prime}\right) \neq 0$, for $|r| \geq 2 \sqrt{K} T, \xi \in \Theta, x^{\prime} \in \mathcal{G}_{k}$, which is true by assumption (ii) of the theorem). We have

$$
\tilde{h}_{k}(r, \xi)=D_{k} u_{k}(r)-K v_{k}(r) .
$$

By part (iii) of Proposition 2.2 we get

$$
\operatorname{Var}_{\left[M_{0}, M_{\xi}\right]}\left(v_{k}\right) \geq 1-(4 K)^{-1},
$$

for any $M_{\xi} \geq 4^{5} 10^{20} K^{5} M_{0}$. 
Using part (v) of Proposition 2.2 we choose

$$
M_{\xi} \in\left[2 \cdot 4^{5} 10^{20} K^{5} M_{0}, 2^{4 \cdot 10^{5} K} 4^{5} 10^{20} K^{5} M_{0}\right] \subset\left(M_{0}, M_{1}\right),
$$

such that

$$
\operatorname{Var}_{\left[M_{0}, M_{\xi}\right]}\left(u_{k}\right) \leq 1+(4 K)^{-1} .
$$

Using (2.1),

$$
\begin{aligned}
\operatorname{Var}_{\left[M_{0}, M_{\xi}\right]}\left(\tilde{h}_{k}(\cdot, \xi)\right) & \geq\left(\operatorname{Var}_{\left[M_{0}, M_{\xi}\right]}^{1 / 2}\left(D_{k} u_{k}\right)-\operatorname{Var}_{\left[M_{0}, M_{\xi}\right]}^{1 / 2}\left(K v_{k}\right)\right)^{2} \\
& \geq\left(K\left(1-(4 K)^{-1}\right)^{1 / 2}-(K-1)\left(1+(4 K)^{-1}\right)^{1 / 2}\right)^{2} \\
& \geq 1 / 4 .
\end{aligned}
$$

Plugging the above estimate in (2.10) yields that

$$
\operatorname{Var}_{\left[M_{0}, M\right]}\left(\tilde{h}_{k}(\cdot, \xi)\right) \geq \frac{M_{0}\left(2 \cdot 4^{5} 10^{20} K^{5}-1\right)}{4 M_{0}\left(2^{10^{6} K}-1\right)} \geq 2^{-10^{6} K} .
$$

This concludes the proof.

\section{Analysis of the determinant and of the minors as polynomials in terms of the potentials}

Let $f_{\Lambda}^{E}=\operatorname{det}\left(H_{\Lambda}-E\right)$ and let $g_{\Lambda}^{E}(i, j)$ be the $(i, j)$ minor of $H_{\Lambda}-E$. In this section we are interested in $f_{\Lambda}^{E}$ and $g_{\Lambda}^{E}(i, j)$ as polynomials in $\left(V_{i}\right)_{i \in \Lambda}$. We will prove Theorem 1.1, as a consequence of Theorem 1.3, and we will provide bounds on the moments of $\Sigma_{\Lambda}^{E}$, which will be needed in Section 4. The properties of $f_{\Lambda}^{E}$ and $g_{\Lambda}^{E}(i, j)$ that are needed for these results are established in the next two propositions.

In the following it is useful to keep in mind that if we order the points of $\mathbb{Z}_{W}$ lexicographically, i.e. $i<j$ if $i_{1}<j_{1}$, or $i_{1}=j_{1}$ and $i_{2}<j_{2}$, then the matrix of $H_{\Lambda}, \Lambda=[a, b]_{W}$, is

$$
\left[\begin{array}{cccccc}
S_{a} & -I & 0 & 0 & \ldots \ldots \ldots \\
-I & S_{a+1} & -I & 0 & \ldots \ldots \ldots \\
\ldots & \ddots & \ddots & \ddots & \ldots \ldots \ldots \\
\ldots \ldots \ldots & \ddots & \ddots & \ddots & \ldots \\
\ldots \ldots & 0 & -I & S_{b-1} & -I \\
\ldots \ldots & \ldots & 0 & 0 & -I & S_{b}
\end{array}\right] .
$$


For the application of Theorem 1.3 we will only need the first part of the following result. The second part will be needed for establishing the Cartan type estimate for $\log \Sigma_{\Lambda}^{E}$ in Lemma 3.3.

Proposition 3.1. Let $i, j \in \Lambda=[a, b]_{W}$ be such that $i_{1}<j_{1}$ and let $n \in\left(i_{1}, j_{1}\right)$.

(i) The degree of $g_{\Lambda}^{E}(i, j)$ as a polynomial of $\left(V_{k}\right)_{k \in\{n\}_{W}}$ is at most $W-1$.

(ii) If $i_{2}=j_{2}$ then the polynomial $\left[g_{\Lambda}^{E}(i, j)\right](V)$ has a monomial whose coefficient is \pm 1 . Furthermore, the degree of $\left[g_{\Lambda}^{E}(i, j)\right](V)$ as a polynomial of $\left(V_{k}\right)_{k \in\{n\}_{W}}$ is $W-1$.

Proof. It is enough to prove the result for $E=0$.

(i) $g_{\Lambda}^{E}(i, j)$ is the determinant of a matrix of the form

$$
\left[\begin{array}{ccc}
* & * & 0 \\
* & S_{n} & * \\
0 & * & *
\end{array}\right]
$$

where the top-right corner entry is a $(p-1) \times(q-1)$ matrix and the lower-left corner entry is a $q \times p$ matrix, with $p=(n-a) W$ and $q=(b-n) W$. The coefficient of the monomial $\prod_{k \in\{n\}_{W}} V_{k}$ is (up to sign) the determinant of the matrix obtained by removing the rows and and the columns corresponding to $S_{n}$. This matrix is of the form

$$
\left[\begin{array}{lll}
* & * & 0 \\
0 & 0 & * \\
0 & 0 & *
\end{array}\right],
$$

where the entries on the diagonal are blocks of size $(p-1) \times(p-1), 1 \times 1$, and $(q-1) \times(q-1)$ respectively. Hence the determinant is zero and the conclusion follows.

(ii) For fixed $i, j \in \Lambda$ let $H_{\Lambda}^{i j}$ be the operator corresponding the matrix obtained from $H_{\Lambda}$ by making all entries on the $i$-th row and on the $j$-th column zero, except for the $(i, j)$-th entry which is set to 1 . Up to sign, $g_{\Lambda}^{E}(i, j)$ is the determinant of $H_{\Lambda}^{i j}$. We will use $h$ to denote the entries of the matrix representation of $H_{\Lambda}^{i j}$. By the Leibniz formula for determinants

$$
g_{\Lambda}^{E}(i, j)=\sum_{\sigma} \operatorname{sgn}(\sigma) \prod_{l \in \Lambda} h_{l, \sigma(l)},
$$

where $\sigma$ runs over all permutations of $\Lambda$. We are interested in the non-zero terms from the above sum that are divisible by $V^{\alpha}$ where $\alpha \in\{0,1\}^{\Lambda}$ and

$$
\alpha_{l}=\left\{\begin{array}{ll}
1 & \text { if } l_{1} \notin\left[i_{1}, j_{1}\right], \text { or } l_{1} \in\left[i_{1}, j_{1}\right] \text { and } l_{2} \neq j_{2} \\
0 & \text { otherwise }
\end{array} .\right.
$$


For each $l$ there are at most $W+2$ values for $\sigma(l)$ such that $h_{l, \sigma(l)}$ is not zero. The permutations $\sigma$ corresponding to non-zero terms divisible by $V^{\alpha}$ must satisfy $\sigma(l)=l$ when $\alpha_{l}=1$. It follows that, for such permutations, $\sigma\left(\left[i_{1}, j_{1}\right] \times\left\{j_{2}\right\}\right)=$ $\left[i_{1}, j_{1}\right] \times\left\{j_{2}\right\}$. Note that by our definition of $H_{\Lambda}^{i j}$ we must have $\sigma\left(i_{1}, j_{2}\right)=\left(j_{1}, j_{2}\right)$. Hence we must have $\sigma\left(\left(i, j_{2}\right)\right)=\left(i-1, j_{2}\right)$, for any $i \in\left(i_{1}, j_{1}\right]$. So $h_{l, \sigma(l)}= \pm 1$, whenever $\alpha_{l}=0$.

This shows that the monomial $V^{\alpha}$ has coefficient \pm 1 . From this it also follows that the degree of $\left[g_{\Lambda}^{E}(i, j)\right](V)$ as a polynomial of $\left(V_{k}\right)_{k \in\{n\}_{W}}$ is at least $W-1$. Now the conclusion follows from part (i).

Remark. The second part of the previous proposition doesn't necessarily hold when $i_{2} \neq j_{2}$. In particular, it can be seen that $g_{\Lambda}^{E}(i, j)$ is identically zero for any $i, j \in \Lambda$, with $i_{2} \neq j_{2}$, provided that $S=0$.

For the next result we will need some bounds on the probability distribution of the resolvent. From [1, Theorem II.1] we have

$$
\mathrm{P}\left(\left|G_{\Lambda}^{E}(i, j)\right| \geq T\right) \lesssim A_{0} / T,
$$

for any $i, j \in \Lambda$. For future use we also note that in our setting the Wegner estimate

$$
\mathbb{P}\left(\left\|G_{\Lambda}^{E}\right\| \geq T\right) \lesssim A_{0}|\Lambda| / T
$$

follows, for example, from [3, (2.4)].

Proposition 3.2. Let $\Lambda_{0}=\{n\}_{W} \subset \Lambda=[a, b]_{W}$. For any

$$
T \geq \max (|E|,\|S\|)
$$

there exists a set $\mathcal{B}=\mathcal{B}(n, T) \subset \mathbb{R}^{\Lambda_{0}^{\prime}}$, with $\mathrm{P}(\mathcal{B}) \lesssim W A_{0} / T$, such that

$$
f_{\Lambda}^{E}\left(V, V^{\prime}\right) \neq 0
$$

for any $V \in \mathbb{C}^{\Lambda_{0}}, \min _{i \in \Lambda_{0}}\left|V_{i}\right| \geq 10 W T, V^{\prime} \in \mathbb{R}^{\Lambda_{0}^{\prime}} \backslash \mathcal{B}$.

Proof. Using (B.1) and Lemma B.1 we have

$$
f_{\Lambda}^{E}=\operatorname{det}\left(H_{\Lambda} / H_{\Lambda_{0}^{\prime}}-E\right) \operatorname{det}\left(H_{\Lambda_{0}^{\prime}}-E\right),
$$

where

$$
H_{\Lambda} / H_{\Lambda_{0}^{\prime}}=H_{\Lambda_{0}}-\Gamma_{0} G_{\Lambda_{0}^{\prime}}^{E} \Gamma_{0}^{*}=\operatorname{diag}\left(V_{(n, 1)}, \ldots, V_{(n, W)}\right)+S-\Gamma_{0} G_{\Lambda_{0}^{\prime}}^{E} \Gamma_{0}^{*}
$$


If $\left|G_{\Lambda_{0}^{\prime}}^{E}(k, l)\right| \leq T$ for any $k, l \in \partial_{\Lambda} \Lambda_{0}$ then $\left|\left(\Gamma_{0} G_{\Lambda_{0}^{\prime}}^{E} \Gamma_{0}^{*}\right)(i, j)\right| \leq 4 T$ for any $i, j \in \Lambda_{0}$, and consequently $\left\|\Gamma_{0} G_{\Lambda_{0}^{\prime}}^{E} \Gamma_{0}^{*}\right\| \leq 4 W T$. Furthermore, if we also have that $\min _{i \in \Lambda_{0}}\left|V_{i}\right| \geq 10 W T$ and $T \geq \max (|E|,\|S\|)$, then $H_{\Lambda} / H_{\Lambda_{0}^{\prime}}-E$ is invertible since

$$
\left\|\operatorname{diag}\left(V_{(n, 1)}, \ldots, V_{(n, W)}\right)^{-1}\right\| \cdot\left\|-E+S-\Gamma_{0} G_{\Lambda_{0}^{\prime}}^{E} \Gamma_{0}^{*}\right\| \leq \frac{6 W T}{10 W T}<1 .
$$

The conclusion follows by setting

$$
\begin{gathered}
\mathcal{B}=\left\{V^{\prime} \in \mathbb{R}^{\Lambda_{0}^{\prime}}:\left|G_{\Lambda_{0}^{\prime}}^{E}(k, l)\right|>T, k, l \in \partial_{\Lambda} \Lambda_{0}\right\} \\
\cup\left\{V^{\prime} \in \mathbb{R}^{\Lambda_{0}^{\prime}}: \operatorname{det}\left(H_{\Lambda_{0}^{\prime}}-E\right)=0\right\} .
\end{gathered}
$$

The bound on $\mathbb{P}(\mathcal{B})$ follows from (3.1).

We can now prove Theorem 1.1

Proof of Theorem 1.1. The result follows by applying Theorem 1.3 with

$$
P(V)=\sum\left|\left[g_{\lambda}^{E}(i, j)\right](V)\right|^{2}, Q(V)=\left|f_{\Lambda}^{E}(V)\right|^{2}, \quad J_{k}=\{k\}_{W}, k \in(a, b) .
$$

Note that $P$ and $Q$ are polynomials of real variables, but with possibly complex coefficients. The assumptions on $P$ and $Q$ are satisfied due to Proposition 3.1 and Proposition 3.2.

To establish the bounds on the moments we need the following Cartan's estimate for Green's function.

Lemma 3.3. There exist absolute constants $C_{0}$ and $C_{1}$ such that for any $R \geq e$ and $H \gg 1$ we have

$$
\operatorname{mes}\left\{V \in \mathbb{R}^{\Lambda}:\|V\| \leq R, \log \Sigma_{\Lambda}^{E} \leq-C_{0} H M_{R}\right\} \leq C_{1}^{|\Lambda|} R^{|\Lambda|} \exp (-H),
$$

where $M_{R}=|\Lambda| \max (1, \log |E|, \log \|S\|) \log R$.

Proof. We have

$$
\left\|H_{\Lambda}^{i j}(V)-E\right\| \leq 1+\left\|H_{\Lambda}(V)-E\right\| \leq 1+|E|+R+\|S\|,
$$

for any $V \in \mathbb{C}^{\Lambda},\|V\| \leq R$, and any $i, j \in \Lambda$ (recall that $H_{\Lambda}^{i j}$ was defined in the proof of Proposition 3.1). Consequently, there exists an absolute constant $B$ such that

$$
\begin{aligned}
\sup _{\|V\| \leq R} \log \left|f_{\Lambda}^{E}(V)\right| & \leq|\Lambda| \log (|E|+R+\|S\|) \\
& \leq B|\Lambda| \max (1, \log |E|, \log \|S\|) \log R
\end{aligned}
$$


and

$$
\begin{aligned}
\sup _{\|V\| \leq R} \log \left|\left[g_{\Lambda}^{E}(i, j)\right](V)\right| & \leq|\Lambda| \log (1+|E|+R+\|S\|) \\
& \leq B|\Lambda| \max (1, \log |E|, \log \|S\|) \log R,
\end{aligned}
$$

for $R \geq e$. Let

$$
M=B|\Lambda| \max (1, \log |E|, \log \|S\|) \log R
$$

and $C_{0}$ as in Lemma A.2. If

$$
\log \Sigma_{\Lambda}^{E} \leq-3 C_{0} H M
$$

then

$$
\begin{aligned}
\log \left|\left[g_{\Lambda}^{E}\left(i^{\prime}, j^{\prime}\right)\right]\right| & \leq \frac{1}{2}\left(\log \Sigma_{\Lambda}^{E}+\log \left|f_{\Lambda}^{E}\right|\right) \\
& \leq-\frac{3}{2} C_{0} H M+\frac{1}{2} \log \left|f_{\Lambda}^{E}\right| \\
& \leq-C_{0} H M,
\end{aligned}
$$

where we chose $i^{\prime} \in\{a\}_{W}$ and $j^{\prime} \in\{b\}_{W}$ (assuming $\Lambda=[a, b]_{W}$ ) such that $i_{2}^{\prime}=j_{2}^{\prime}$. The conclusion follows by applying Lemma A.2 to $\log \left|\left[g_{\Lambda}^{E}\left(i^{\prime}, j^{\prime}\right)\right]\right|$. This is possible due to Proposition 3.1 (ii). Note that the constant $C_{0}$ from the result is not the same as in Lemma A.2.

Proposition 3.4. Given $s \geq 1$ there exists a constant

$$
C_{0}=C_{0}\left(A_{0}, A_{1},|E|, s,\|S\|\right)
$$

such that

$$
\mathbb{E}\left(\log ^{s} \Sigma_{\Lambda}^{E}\right) \leq C_{0}(|\Lambda| \log |\Lambda|)^{2 s}, \quad|\Lambda|>1 .
$$

Proof. From Lemma 3.3 and Lemma A.3 it follows that for any $R \geq e$ we have

$$
\int_{\|V\| \leq R} \log ^{s} \Sigma_{\Lambda}^{E} d v \leq\left(C|\Lambda|^{2} \log ^{2} R\right)^{s},
$$

with $C=C\left(A_{0},|E|,\|S\|\right)$. 
Note that due to (1.2) we have

$$
\mathbb{P}(\|V\| \geq R) \leq \sum_{i \in \Lambda} \mathbb{P}\left(\left|V_{i}\right| \geq R /|\Lambda|^{1 / 2}\right) \leq A_{1}|\Lambda|^{3 / 2} / R
$$

Let $R_{k}=R_{0}^{k}|\Lambda|^{3 / 2}$, with $R_{0} \gg e$. Using the two previous estimates we have

$$
\begin{aligned}
& \mathbb{E}\left(\log ^{s} \Sigma_{\Lambda}^{E}\right) \\
& \quad=\int_{\|V\| \leq R_{1}} \log ^{s} \Sigma_{\Lambda}^{E} d v+\sum_{k=1}^{\infty} \int_{R_{k}<\|V\| \leq R_{k+1}} \log ^{s} \Sigma_{\Lambda}^{E} d v \\
& \quad \leq\left(C|\Lambda|^{2} \log ^{2} R_{1}\right)^{s}+\sum_{k=1}^{\infty}\left(\int_{\|x\| \leq R_{k+1}} \log ^{2 s} \Sigma_{\Lambda}^{E} d v\right)^{1 / 2}\left(\mathbb{P}\left(\|V\| \geq R_{k}\right)\right)^{1 / 2} \\
& \quad \leq(C|\Lambda| \log |\Lambda|)^{2 s}+(C|\Lambda| \log |\Lambda|)^{2 s} \sum_{k=1}^{\infty}\left(\log ^{2} R_{0}^{k+1}\right)^{s}\left(A_{1} / R_{0}^{k}\right)^{1 / 2} \\
& \quad \leq C(s)(|\Lambda| \log |\Lambda|)^{2 s} .
\end{aligned}
$$

\section{Large Fluctuations Imply Exponential Decay}

In this section we show how to pass from fluctuations of the resolvent to exponential decay. The main result is Theorem 4.4. The basic idea, developed in Proposition 4.1, is that having some fluctuations of Green's function implies some exponential decay with non-zero probability. The desired result will follow by standard multi-scale analysis. The initial estimate is provided in Proposition 4.2 and the inductive step is implemented in Proposition 4.3 (cf. [9, Lemma 4.1]). Throughout this section we assume

$$
\operatorname{Var}\left(\log \Sigma_{\Lambda}^{E}\right) \geq L \delta_{0}
$$

with $\delta_{0} \leq 1 / W$, for any $\Lambda=[a, b]_{W}, b-a+1=L$.

Proposition 4.1. Given $\varepsilon \in(0,1)$ there exists $C_{0}=C_{0}\left(A_{0}, A_{1}, \varepsilon,|E|,\|S\|\right)$ such that

$$
\mathrm{P}\left(\log \Sigma_{\Lambda}^{E} \leq-\sqrt{L \delta_{0}} / 2\right) \geq\left(\frac{L \delta_{0}}{C_{0}|\Lambda|^{4} \log ^{4}|\Lambda|}\right)^{1+\varepsilon},
$$

for any $\Lambda=[a, b]_{W}, b-a+1=L \geq C_{0} \delta_{0}^{-1} \log ^{2} \delta_{0}$. 
Proof. We partition $\mathbb{R}^{\Lambda}$ by the sets

$$
\begin{aligned}
\Omega_{-1} & =\left\{V: \log \Sigma_{\Lambda}^{E} \leq-\sqrt{L \delta_{0}} / 2\right\}, \\
\Omega_{0} & =\left\{V:\left|\log \Sigma_{\Lambda}^{E}\right|<\sqrt{L \delta_{0}} / 2\right\}, \\
\Omega_{1} & =\left\{V: \log \Sigma_{\Lambda}^{E} \geq \sqrt{L \delta_{0}} / 2\right\} .
\end{aligned}
$$

By our assumption on the variance we have that $\mathbb{E}\left(\log ^{2} \Sigma_{\Lambda}^{E}\right) \geq L \delta_{0}$. At the same time we have both

$$
\int_{\Omega_{0}} \log ^{2} \Sigma_{\Lambda}^{E} d \nu \leq L \delta_{0} / 4
$$

and

$$
\begin{aligned}
\int_{\Omega_{-1}} \log ^{2} \Sigma_{\Lambda}^{E} d \nu & \leq\left(\int_{\mathbb{R}^{\Lambda}} \log ^{2(1+\varepsilon) / \varepsilon} \Sigma_{\Lambda}^{E} d \nu\right)^{\varepsilon /(1+\varepsilon)}\left(\mathbb{P}\left(V \in \Omega_{-1}\right)\right)^{1 /(1+\varepsilon)} \\
& \leq C|\Lambda|^{4} \log ^{4}|\Lambda|\left(\mathbb{P}\left(V \in \Omega_{-1}\right)\right)^{1 /(1+\varepsilon)}
\end{aligned}
$$

as well as

$$
\begin{aligned}
\int_{\Omega_{1}} \log ^{2} \Sigma_{\Lambda}^{E} d \nu & \leq\left(\int_{\mathbb{R}^{\Lambda}} \log ^{4} \Sigma_{\Lambda}^{E} d \nu\right)^{1 / 2}\left(\mathbb{P}\left(V \in \Omega_{1}\right)\right)^{1 / 2} \\
& \leq C|\Lambda|^{4} \log ^{4}|\Lambda|\left(\mathbb{P}\left(V \in \Omega_{1}\right)\right)^{1 / 2}
\end{aligned}
$$

with $C=C\left(A_{0}, A_{1}, \varepsilon,|E|,\|S\|\right)$, due to Proposition 3.4. We conclude that

$$
\begin{aligned}
& \mathbb{P}\left(\log \Sigma_{\Lambda}^{E} \leq-\sqrt{L \delta_{0}} / 2\right) \\
& \quad \geq\left(\frac{3 L \delta_{0} / 4-C|\Lambda|^{4} \log ^{4}|\Lambda|\left(\mathbb{P}\left(\log \Sigma_{\Lambda}^{E} \geq \sqrt{L \delta_{0}} / 2\right)\right)^{1 / 2}}{C|\Lambda|^{4} \log ^{4}|\Lambda|}\right)^{1+\varepsilon} .
\end{aligned}
$$

Now we just need to estimate the probability on the right-hand side. If $\log \Sigma_{\Lambda}^{E} \geq$ $\sqrt{L \delta_{0}} / 2$ then $\left|G_{\Lambda}^{E}(i, j)\right| \geq \exp \left(\sqrt{L \delta_{0}} / 2\right) / W^{2}$ for some $(i, j) \in \partial \Lambda, i_{1}<j_{1}$. Using the estimate (3.1) we have

$$
\mathrm{P}\left(\log \Sigma_{\Lambda}^{E} \geq \sqrt{L \delta_{0}} / 2\right) \lesssim A_{0} W^{4} \exp \left(-\sqrt{L \delta_{0}} / 2\right) .
$$

The conclusion follows because

$$
3 L \delta_{0} / 4-C|\Lambda|^{4} \log ^{4}|\Lambda|\left(A_{0} W^{4} \exp \left(-\sqrt{L \delta_{0}} / 2\right)\right)^{1 / 2} \geq L \delta_{0} / 4,
$$

for $L \geq C^{\prime} \delta_{0}^{-1} \log ^{2} \delta_{0}$ (recall that we are assuming $\delta_{0} \leq W^{-1}$ ). 
Proposition 4.2. Fix $\beta \geq 1$. There exists $C_{0}=C_{0}\left(A_{0}, A_{1}, \beta,|E|,\|S\|\right)$ such that

$$
\mathbb{P}\left(\log \left|G_{\Lambda_{L}(a)}^{E}(i, j)\right| \leq-\frac{\delta_{0}^{1 / 2} L^{1 / 10}}{4}, i \in\{a\}_{W}, j \in \partial \Lambda_{L}(a)\right) \geq 1-L^{-\beta},
$$

for any $L \geq C_{0} \delta_{0}^{-6} W^{20}$.

Proof. We only prove that

$$
\mathbb{P}\left(\log \left|G_{\Lambda_{L}(a)}^{E}(i, j)\right| \leq-\frac{\delta_{0}^{1 / 2} L^{1 / 10}}{4}, i \in\{a-L\}_{W}, j \in\{a\}_{W}\right) \geq 1-\frac{L^{-\beta}}{2} .
$$

The same estimate with $i \in\{a\}_{W}$ and $j \in\{a+L\}_{W}$ will hold by an analogous proof.

Let $l=\left[L^{1 / 5}\right]$. We have $l^{5} \leq L<2 l^{5}$ (provided $L$ is larger than some absolute constant). Let $\mathcal{G}_{1}$ be the event that $\log \Sigma_{\Lambda_{0}}^{E} \leq-\sqrt{l \delta_{0}} / 2$ holds for at least one block

$$
\Lambda_{0}=[n l+1,(n+1) l]_{W} \subset \Lambda=[a-L, a]_{W} .
$$

Clearly $\Lambda$ contains more than $l^{4} / 2$ such blocks. By the independence of the potentials and by Proposition 4.1 we have that for $\varepsilon$ small enough

$$
\begin{aligned}
\mathbb{P} & \left.\mathbb{R}^{\Lambda} \backslash \mathcal{G}_{1}\right) \\
& \leq\left(1-c\left(\delta_{0} l\right)^{1+\varepsilon} /(l W)^{4(1+2 \varepsilon)}\right)^{l^{4} / 2} \\
& \leq \exp \left(-c\left(\delta_{0} l\right)^{1+\varepsilon} /(l W)^{4(1+2 \varepsilon)} l^{4}\right) \\
& \leq \exp \left(-c \delta_{0}^{1+\varepsilon} W^{-4(1+2 \varepsilon)} L^{(1-7 \varepsilon) / 5}\right) \\
& \leq L^{-\beta} / 4,
\end{aligned}
$$

provided that $L \geq C \delta_{0}^{-6} W^{20}$. Let $\mathcal{G}_{2}$ be the event that $\left\|G_{\Lambda_{L}(a)}^{E}\right\| \leq T$ and $\left\|G_{\Lambda_{1}}^{E}\right\| \leq T$ for any

$$
\Lambda_{1}=[a-L,(n+1) l]_{W} \subset \Lambda,
$$

with $T \geq 1$ to be chosen later. From (3.2) it follows that

$$
\mathbb{P}\left(\mathbb{R}^{\Lambda} \backslash \mathcal{G}_{2}\right) \lesssim A_{0} L^{2} W T^{-1} .
$$


For the event $\mathcal{G}_{1} \cap \mathcal{G}_{2}$ it follows, by using the second resolvent identity (B.3), that

$$
\begin{aligned}
\left|G_{\Lambda_{L}(a)}^{E}(i, j)\right| & =\left|\sum_{\left(k, k^{\prime}\right) \in \partial_{\Lambda_{L}(a)} \Lambda_{1}} G_{\Lambda_{1}}^{E}(i, k) G_{\Lambda_{L}(a)}^{E}\left(k^{\prime}, j\right)\right| \\
& \leq T W\left|G_{\Lambda_{1}}^{E}(i, \tilde{k})\right| \\
& =T W\left|\sum_{\left(l, l^{\prime}\right) \in \partial_{\Lambda_{1}} \Lambda_{0}} G_{\Lambda_{0}}^{E}(\tilde{k}, l) G_{\Lambda_{1}}^{E}\left(l^{\prime}, i\right)\right| \\
& \leq T W \exp \left(-\sqrt{l \delta_{0}} / 4\right)\left|G_{\Lambda_{1}}^{E}(\tilde{l}, i)\right| \\
& \leq T^{2} W \exp \left(-\sqrt{l \delta_{0}} / 4\right) \leq \exp \left(-\delta_{0}^{1 / 2} L^{1 / 10} / 8\right),
\end{aligned}
$$

provided

$$
T=\exp \left(\frac{\delta_{0}^{1 / 2} L^{1 / 10}}{16}\right) \quad \text { and } \quad L \geq C \delta_{0}^{-5} \log ^{10} W .
$$

The conclusion follows by noticing that with this choice of $T$ we have

$$
A_{0} L^{2} W T^{-1} \leq L^{-\beta} / 4,
$$

for $L \geq C \delta_{0}^{-5} \log ^{10} W$.

Proposition 4.3. Fix $\beta \geq 1$ and $\varepsilon \in(0,1)$. There exists a constant

$$
C_{0}=C_{0}\left(\beta, \varepsilon, A_{0}\right)
$$

such that if, for some $l \geq C_{0}$,

$$
\mathbb{P}\left(\log \left|G_{\Lambda_{l}(a)}^{E}(i, j)\right| \leq-m_{l} l, i \in\{a\}_{W}, j \in \partial \Lambda_{l}(a)\right) \geq 1-l^{-\beta},
$$

with $m_{l} \geq l^{\varepsilon-1} \log W$, for any $\Lambda_{l}(a) \subset \mathbb{Z}_{W}$, then, for $L=l^{\alpha}, \alpha \in[2,4]$, and any $\Lambda_{L}(a) \subset \mathbb{Z}_{W}$,

$$
\mathrm{P}\left(\log \left|G_{\Lambda_{L}(a)}^{E}(i, j)\right| \leq-m_{L} L, i \in\{a\}_{W}, j \in \partial \Lambda_{L}(a)\right) \geq 1-L^{-\beta},
$$

with

$$
m_{l} \geq m_{L} \geq\left(1-6 l^{-1 / 4}\right) m_{l}-\log (2 W) / l \geq L^{\varepsilon-1} \log W .
$$


Proof. Let

$$
I=[a-L+l, a+L-l] .
$$

We say that $b \in I$ is good if

$$
\log \left|G_{\Lambda_{l}(b)}^{E}(i, j)\right| \leq-m_{l} l, i \in\{b\}_{W}, j \in \partial \Lambda_{l}(b) .
$$

We partition $I$ into $2 l+1$ subsets

$$
I_{s}=\{b \in I: b=s(\bmod 2 l+1)\} .
$$

For each $s$ the set $I_{s}$ has at least

$$
n=\frac{2 L-4 l+1}{2 l+1}-1
$$

elements and the blocks $\Lambda_{l}(b), b \in I_{s}$ are disjoint. By Hoeffding's inequality (see [5, Theorem 1]) applied to the binomial distribution with parameters $n$ and $p=1-l^{-\beta}$ we have that there exist at least $(1-\delta) p n \operatorname{good} b$ 's in $I_{s}$, with probability greater than $1-\exp \left(-2(p n-(1-\delta) p n)^{2} / n\right)$. Let $B$ be the number of bad $u \in I$. By choosing $\delta=l^{-1 / 4}$ it follows that

$$
\begin{aligned}
B & \leq 2 L-2 l+1-(2 l+1)(1-\delta) p n \\
& =(2 L-2 l+1)[1-(1-\delta) p]+(4 l+1)(1-\delta) p \\
& \leq 4 L l^{-1 / 4},
\end{aligned}
$$

with probability greater than

$$
\begin{aligned}
1-(2 l+1) \exp \left(-2 n p^{2} \delta^{2}\right) & \geq 1-(2 l+1) \exp \left(-c L \delta^{2} / l\right) \\
& \geq 1-(2 l+1) \exp \left(-c l^{1 / 2}\right) \\
& \geq 1-L^{-\beta} / 2,
\end{aligned}
$$

provided that $l \geq C=C(\beta)$.

Let $\Lambda_{t}$ be the blocks corresponding to the connected components of the set of bad elements in $I$. Clearly $t \leq B$ and if $l_{t}$ is the length of $\Lambda_{t}$ then $\sum l_{t}=B$. Using (3.2) we know that with probability greater than $1-C A_{0} W L^{3} T^{-1}$ we have $\left\|G_{\Lambda}^{E}\right\| \leq T$, where $\Lambda$ is any of the blocks $\Lambda_{t}$ or $\Lambda_{L}(a)$. We will choose $T$ later.

Let $i \in\{a\}_{W}$ and $j \in \partial \Lambda_{L}(a)$. We will use the resolvent identity (B.3). If $a$ is good then

$$
\begin{aligned}
\left|G_{\Lambda_{L}(a)}^{E}(i, j)\right| & =\left|\sum_{\left(k, k^{\prime}\right) \in \partial_{\Lambda_{L}(a)} \Lambda_{l}(a)} G_{\Lambda_{l}(a)}^{E}(i, k) G_{\Lambda_{L}(a)}^{E}\left(k^{\prime}, j\right)\right| \\
& \leq 2 W \exp \left(-m_{l} l\right)\left|G_{\Lambda_{L}(a)}^{E}(\tilde{k}, j)\right|,
\end{aligned}
$$


for some $\tilde{k} \in \partial_{\Lambda_{L}(a)} \Lambda_{l}(a)$. If $a$ is bad then $\{a\}_{W} \subset \Lambda_{t}$ and by our choice of $\Lambda_{t}$ we know that $\tilde{k}_{1}$ is good for any $\tilde{k} \in \partial_{\Lambda_{L}(a)} \Lambda_{t}$ (provided $k_{1} \in I$ ). So if $a$ is bad we have

$$
\begin{aligned}
\left|G_{\Lambda_{L}(a)}^{E}(i, j)\right| & =\left|\sum_{\left(k, k^{\prime}\right) \in \partial_{\Lambda_{L}(a)} \Lambda_{t}} G_{\Lambda_{t}}^{E}(i, k) G_{\Lambda_{L}(a)}^{E}\left(k^{\prime}, j\right)\right| \\
& \leq 2 W T\left|G_{\Lambda_{L}(a)}^{E}(\tilde{k}, j)\right| \\
& =2 W T\left|\sum_{\left(l, l^{\prime}\right) \in \partial_{\Lambda_{L}(a)} \Lambda_{l}\left(\tilde{k}_{1}\right)} G_{\Lambda_{l}\left(\tilde{k}_{1}\right)}^{E}(\tilde{k}, l) G_{\Lambda_{L}(a)}^{E}\left(l^{\prime}, j\right)\right| \\
& \leq 4 W^{2} T \exp \left(-m_{l} l\right)\left|G_{\Lambda_{L}(a)}^{E}(\tilde{l}, j)\right| \\
& =\left|G_{\Lambda_{L}(a)}^{E}(\tilde{l}, j)\right|,
\end{aligned}
$$

where we chose

$$
T=\frac{\exp \left(m_{l} l\right)}{4 W^{2}} .
$$

We can iterate these estimates as long as $\tilde{k}_{1}, \tilde{J}_{1} \in I$. We conclude that

$$
\left|G_{\Lambda_{L}(a)}^{E}(i, j)\right| \leq T\left(2 W \exp \left(-m_{l} l\right)\right)^{n_{1}} \leq\left(2 W \exp \left(-m_{l} l\right)\right)^{n_{1}-2},
$$

with

$$
n_{1} \geq \frac{L-l+1-B}{l+1}-1
$$

So we have

$$
\begin{aligned}
m_{L}=\frac{n_{1}-2}{L}\left(m_{l} l-\log (2 W)\right) & \geq \frac{1-5 l^{-1 / 4}}{l+1}\left(m_{l} l-\log (2 W)\right) \\
& \geq\left(1-6 l^{-1 / 4}\right) m_{l}-\frac{\log (2 W)}{l},
\end{aligned}
$$

for $l \geq C$. The conclusion follows by noting that

$$
\begin{aligned}
1-C A_{0} W L^{3} T^{-1} & =1-C A_{0} W^{3} L^{3} \exp \left(-m_{l} l\right) \\
& \geq 1-C A_{0} W^{3} L^{3} \exp \left(-l^{\varepsilon} \log W\right) \\
& \geq 1-L^{-\beta} / 2,
\end{aligned}
$$

provided $l \geq C=C\left(\beta, \varepsilon, A_{0}\right)$. 
Theorem 4.4. Fix $\beta \geq 1$. If we have $\operatorname{Var}\left(\Sigma_{\Lambda}^{E}\right) \geq L \delta_{0}$, with $\delta_{0} \leq W^{-1}$, for any $\Lambda=[a, b]_{W}$, with $b-a+1=L$, then there exists

$$
C_{0}=C_{0}\left(A_{0}, A_{1}, \beta,|E|,\|S\|\right)
$$

such that

$$
\mathbb{P}\left(\log \left|G_{\Lambda_{L}(a)}^{E}(i, j)\right| \leq-C_{0}^{-1} \delta_{0}^{6} W^{-20} L, i \in\{a\}_{W}, j \in \partial \Lambda_{L}(a)\right) \geq 1-L^{-\beta},
$$

for any $L \geq C_{0} \delta_{0}^{-12} W^{40}$ and $a \in \mathbb{Z}$.

Proof. Let

$$
L_{0}=B \delta_{0}^{-6} W^{20} \text {. }
$$

If $B$ is large enough, as in Proposition 4.2, then

$$
\mathbb{P}\left(\log \left|G_{\Lambda_{L_{0}}(a)}^{E}(i, j)\right| \leq-m_{L_{0}} L_{0}, i \in\{a\}_{W}, j \in \partial \Lambda_{L_{0}}(a)\right) \geq 1-L_{0}^{-\beta},
$$

with

$$
m_{L_{0}}=\frac{\delta_{0}^{1 / 2} L_{0}^{1 / 10}}{4 L_{0}}=B^{-9 / 10} \frac{\delta_{0}^{59 / 10} W^{18}}{4}
$$

Note that

$$
m_{L_{0}} \geq L_{0}^{1 / 100-1} \log W
$$

provided $B$ is large enough.

Given $L \geq L_{0}^{2}$ we can find a sequence $L_{k}$ such that

$$
L_{k+1}=L_{k}^{\alpha_{k}}, \quad \alpha_{k} \in[2,4]
$$

and

$$
L=L_{k_{0}} \quad \text { for some } k_{0} \geq 1 \text {. }
$$

Applying Proposition 4.3 inductively,

$$
m_{L_{k+1}} \geq\left(1-L_{k}^{-1 / 4}\right) m_{L_{k}}-\frac{\log (2 W)}{L_{k}} .
$$

Consequently we get

$$
m_{L}-m_{L_{0}} \geq-\sum_{k=0}^{\infty}\left(m_{L_{k}} L_{k}^{-1 / 4}+\log (2 W) L_{k}^{-1}\right) \geq-\frac{m_{L_{0}}}{2},
$$

provided that $B$ is large enough (we used the fact that $m_{L_{0}} \geq m_{L_{k}}$ and that $\left.m_{L_{0}} \geq L_{0}^{1 / 100-1} \log W\right)$. The conclusion follows immediately. 


\section{A. Cartan's Estimate}

For convenience we include a statement of the Cartan estimate for analytic functions (see [6, Theorem 11.4]).

Lemma A.1. Let $\phi: \mathbb{D} \rightarrow \mathbb{C}$ be an analytic function such that

$$
m \leq \log |\phi(0)|, M \geq \sup _{\zeta \in \mathbb{D}} \log |\phi(\zeta)|
$$

Then there exists an absolute constant $C_{0}$ such that for any $H \gg 1$ we have

$$
\log |\phi(\zeta)|>M-C_{0} H(M-m)
$$

for all $\zeta \in \mathbb{D}_{1 / 6}$ except for a set of disks with the sum of the radii less than $\exp (-H)$.

The next result is a Cartan type estimate for multivariate polynomials.

Lemma A.2. If $P(x)=\sum_{|\alpha| \leq D} a_{\alpha} x^{\alpha}$ is a polynomial of $N$ variables such that $\max _{|\alpha| \leq D}\left|a_{\alpha}\right| \geq 1$ and $\sup _{\|z\| \leq 20 R_{0}} \log |P(z)| \leq M_{R_{0}}$, for some $R_{0} \geq 1$, then there exist absolute constants $C_{0}$ and $C_{1}$ such that for any $H \gg 1$ we have

$$
\operatorname{mes}\left\{x \in \mathbb{R}^{N}:\|x\| \leq R_{0}, \log |P(x)| \leq-C_{0} H M_{R_{0}}\right\} \leq C_{1}^{N} R_{0}^{N} \exp (-H) .
$$

Proof. The strategy is to apply the one dimensional Cartan's estimate on complex lines that will cover the set $\left\{\|x\| \leq R_{0}\right\}$. For this we need to find a point $x_{0} \in \mathbb{R}^{N}$ at which $\left|P\left(x_{0}\right)\right|$ is bounded away from zero. Due to the Cauchy estimates for the derivatives of analytic functions one has

$$
\left|a_{\alpha}\right| \leq \max _{\|z\| \leq 1}|P(z)|
$$

for any $\alpha$. It follows that there exists $z_{0} \in \mathbb{C}^{N},\left\|z_{0}\right\| \leq 1$, such that $\left|P\left(z_{0}\right)\right| \geq 1$. We will use Cartan's estimate "centered" at $z_{0}$ to show the existence of $x_{0}$. Let $\phi(\zeta)=P\left(z_{0}-10 \zeta \operatorname{Im} z_{0}\right)$. This peculiar definition is motivated by the fact that $z_{0}-10 \zeta \operatorname{Im} z_{0} \in \mathbb{R}^{N}$ whenever $\operatorname{Im} \zeta=1 / 10$. We have that $\log |\phi(0)| \geq 0$ and $\sup _{\zeta \in \mathbb{D}} \log |\phi(\zeta)| \leq M_{R_{0}}$, so Cartan's estimate guarantees, in particular, that there exists $\left|\zeta_{0}\right| \leq 1 / 6$ with $\operatorname{Im} \zeta_{0}=1 / 10$ such that

$$
\log \left|\phi\left(\zeta_{0}\right)\right| \geq-C M_{R_{0}}
$$

with $C \gg 1$. We can now choose $x_{0}=z_{0}-10 \zeta_{0} \operatorname{Im} z_{0}$. 
Let

$$
f(z)=P\left(x_{0}+12 R_{0} z\right) .
$$

We have both

$$
\log |f(0)| \geq-C M_{R_{0}},
$$

and

$$
\sup _{\|z\| \leq 1} \log |f(z)| \leq \sup _{\|z\| \leq 20 R_{0}} \log |P(z)| \leq M_{R_{0}}
$$

as well as

$$
\begin{aligned}
\left\{x \in \mathbb{R}^{N}:\|x\|\right. & \left.\leq R_{0}, \log |P(x)| \leq-C H M_{R_{0}}\right\} \\
& \subset x_{0}+12 R_{0} \underbrace{\left\{x \in \mathbb{R}^{N}:\|x\| \leq 1 / 6, \log |f(x)| \leq-C H M_{R_{0}}\right\}}_{:=\mathcal{B}} .
\end{aligned}
$$

Let $\xi_{0} \in\left\{x \in \mathbb{R}^{N}:\|x\|=1\right\}$. By applying Cartan's estimate to

$$
\varphi(\zeta)=\log \left|f\left(\zeta \xi_{0}\right)\right|
$$

we get $\int_{\mathbb{R}} \mathbb{1}_{\mathcal{B}}\left(r x_{0}\right) d r \leq C \exp (-H)$. The conclusion now follows by integrating $\mathbb{1}_{\mathcal{B}}$ in hyper-spherical coordinates.

We also illustrate how to obtain explicit integrability estimates for functions satisfying a Cartan type estimate.

Lemma A.3. Let $f$ be a measurable function on $\left\{x \in \mathbb{R}^{N}:\|x\| \leq R_{0}\right\}, R_{0}>0$ such that

$$
\operatorname{mes}\left\{x \in \mathbb{R}^{N}:\|x\| \leq R_{0}, \log |f(x)| \leq-C_{0} H M_{0}\right\} \leq C_{1}^{N} R_{0}^{N} \exp (-H),
$$

for some $M_{0} \geq \sup _{\|x\| \leq R_{0}} \log |f(x)|$, and some absolute constants $C_{0}, C_{1}$. Given $s>0$ there exists an absolute constant $C_{2}$ such that if $\mu$ is a probability measure with $d \mu \leq B_{0}^{N} d m$ for some $B_{0}>0$, then

$$
\int_{\|x\| \leq R_{0}}|\log | f(x) \|^{s} d \mu(x) \leq\left(C_{2} M_{0} N \max \left(1, \log B_{0}, \log R_{0}\right)\right)^{s}, s \geq 1
$$


Proof. We have

$$
\begin{aligned}
& \int_{\|x\| \leq R_{0}}|\log | f(x) \|^{s} d \mu(x) \\
&=\int_{0}^{\infty} \mu\left(|\log | f(x)\left\|^{s} \geq \lambda,\right\| x \| \leq R_{0}\right) d \lambda \\
&=\int_{0}^{H_{0}} \mu\left(\left.|\log | f(x)\right|^{s} \geq\left(C H M_{0}\right)^{s},\|x\| \leq R_{0}\right) s C^{s} M_{0}^{s} H^{s-1} d H \\
& \quad+\int_{H_{0}}^{\infty} \mu\left(\log |f(x)| \leq-C H M_{0},\|x\| \leq R_{0}\right) s C^{s} M_{0}^{s} H^{s-1} d H \\
& \leq\left(C M_{0} H_{0}\right)^{s} \\
&+C^{s} M_{0}^{s} B_{0}^{N} \int_{H_{0}}^{\infty} \operatorname{mes}\left\{\log |f(x)| \leq-C H M_{0},\|x\| \leq R_{0}\right\} s H^{s-1} d H \\
& \leq\left(C M_{0} H_{0}\right)^{s}+C^{N+s} M_{0}^{s} B_{0}^{N} R_{0}^{N} \exp \left(-H_{0} / 2\right) \\
& \leq C^{s} M_{0}^{s} N^{s}\left(\max \left(1, \log B_{0}, \log R_{0}\right)\right)^{s} .
\end{aligned}
$$

Note that we chose $H_{0}=C N \max \left(1, \log B_{0}, \log R_{0}\right)$.

\section{B. Resolvent Identities}

Recall the following fundamental facts regarding Schur's complement (see, for example, [10, Theorem 1.1-2]).

Lemma B.1. Let

$$
H=\left[\begin{array}{cc}
H_{0} & \Gamma_{0} \\
\Gamma_{1} & H_{1}
\end{array}\right],
$$

where $H_{0}$ is a $n_{0} \times n_{0}$ matrix and $H_{1}$ is an invertible $n_{1} \times n_{1}$ matrix. Let

$$
H / H_{1}=H_{0}-\Gamma_{0} H_{1}^{-1} \Gamma_{1} .
$$

Then

$$
\operatorname{det} H=\left(\operatorname{det} H / H_{1}\right)\left(\operatorname{det} H_{1}\right)
$$

and if $\mathrm{H} / \mathrm{H}_{1}$ is invertible then

$$
H^{-1}=\left[\begin{array}{cc}
\left(H / H_{1}\right)^{-1} & -\left(H / H_{1}\right)^{-1} \Gamma_{0} H_{1}^{-1} \\
-H_{1}^{-1} \Gamma_{1}\left(H / H_{1}\right)^{-1} & H_{1}^{-1}+H_{1}^{-1} \Gamma_{1}\left(H / H_{1}\right)^{-1} \Gamma_{0} H_{1}^{-1}
\end{array}\right] .
$$


Next we set things up so that we can apply the previous lemma to our finite volume matrices. Let $\Lambda=[a, b] \times[1, W]$ and $\Lambda_{0}=\left[a_{0}, b_{0}\right] \times[1, W]$ be so that $\Lambda_{0} \subset \Lambda$, and let $\Lambda_{0}^{\prime}=\Lambda \backslash \Lambda_{0}$. By viewing $\mathbb{C}^{\Lambda}$ as $\mathbb{C}^{\Lambda_{0}} \oplus \mathbb{C}^{\Lambda_{0}^{\prime}}$ one has the following matrix representation

$$
H_{\Lambda}=\left[\begin{array}{cc}
H_{\Lambda_{0}} & \Gamma_{0} \\
\Gamma_{0}^{*} & H_{\Lambda_{0}^{\prime}}
\end{array}\right]
$$

where

$$
\Gamma_{0}(i, j)= \begin{cases}-1 & \text { if }\left|i_{1}-j_{1}\right|=1 \text { and } i_{2}=j_{2}, \\ 0 & \text { otherwise }\end{cases}
$$

(note that, implicitly, $i \in \Lambda_{0}$ and $j \in \Lambda_{0}^{\prime}$ ).

We recall the second resolvent identity (see, for example, [8, Lemma 6.5]) as used in $[4,(2.12)]$. We have that

$$
H_{\Lambda}=H_{\Lambda_{0}} \oplus H_{\Lambda_{0}^{\prime}}+\Gamma,
$$

with

$$
\Gamma=\left[\begin{array}{cc}
0 & \Gamma_{0} \\
\Gamma_{0}^{*} & 0
\end{array}\right]
$$

The second resolvent identity gives us that

$$
G_{\Lambda}^{E}=G_{\oplus}^{E}-G_{\oplus}^{E} \Gamma G_{\Lambda}^{E},
$$

where $G_{\oplus}^{E}=G_{\Lambda_{0}}^{E} \oplus G_{\Lambda_{0}^{\prime}}^{E}$. We have that

$$
\Gamma(i, j)= \begin{cases}-1 & \text { if }(i, j) \in \partial_{\Lambda} \Lambda_{0} \text { or }(j, i) \in \partial_{\Lambda} \Lambda_{0}, \\ 0 & \text { otherwise. }\end{cases}
$$

It follows that, for any $i \in \Lambda_{0}$ and $j \in \Lambda_{0}^{\prime}$,

$$
G_{\Lambda}^{E}(i, j)=\sum_{\left(k, k^{\prime}\right) \in \partial_{\Lambda} \Lambda_{0}} G_{\Lambda_{0}}^{E}(i, k) G_{\Lambda}^{E}\left(k^{\prime}, j\right) .
$$

\section{References}

[1] M. Aizenman and S. Molchanov, Localization at large disorder and at extreme energies: an elementary derivation. Comm. Math. Phys. 157 (1993), no. 2, 245-278. MR 1244867 Zbl 0782.60044

[2] J. Bourgain, A lower bound for the Lyapounov exponents of the random Schrödinger operator on a strip. J. Stat. Phys. 153 (2013), no. 1, 1-9. MR 3100812 Zbl 06235201 
[3] J.-M. Combes, F. Germinet, and A. Klein, Generalized eigenvalue-counting estimates for the Anderson model. J. Stat. Phys. 135 (2009), no. 2, 201-216. MR 2505733 Zbl 1168.82016

[4] J. Fröhlich and Th. Spencer, Absence of diffusion in the Anderson tight binding model for large disorder or low energy. Comm. Math. Phys. 88 (1983), no. 2, 151-184. MR 696803 Zbl 0519.60066

[5] W. Hoeffding, Probability inequalities for sums of bounded random variables. J. Amer. Statist. Assoc. 58 (1963), 13-30. MR 0144363 Zbl 0127.10602

[6] B. Ya. Levin, Lectures on entire functions. Translations of Mathematical Monographs, vol. 150. American Mathematical Society, Providence, R.I., 1996. In collaboration with and with a preface by Yu. Lyubarskii, M. Sodin, and V. Tkachenko. Translated from the Russian manuscript by V. Tkachenko. MR 1400006 Zbl 0856.30001

[7] J. Schenker, Eigenvector localization for random band matrices with power law band width. Comm. Math. Phys. 290 (2009), no. 3, 1065-1097. MR 2525652 Zbl 1179.82079

[8] G. Teschl, Mathematical methods in quantum mechanics. With applications to Schrödinger operators. Graduate Studies in Mathematics, 99. American Mathematical Society, Providence, R.I., 2009. MR 2499016 Zbl 1166.81004

[9] H. von Dreifus and A. Klein, A new proof of localization in the Anderson tight binding model. Comm. Math. Phys. 124 (1989), no. 2, 285-299. MR 1012868 Zbl 0698.60051

[10] F. Zhang (ed.), The Schur complement and its applications. Numerical Methods and Algorithms, 4. Springer, New York, 2005. MR 2160825 Zbl 1075.15002

Received October 4, 2013; revised April 28, 2014

Ilia Binder, Department of Mathematics, University of Toronto, Toronto, ON, M5S 2E4, Canada

e-mail: ilia@math.utoronto.ca

Michael Goldstein, Department of Mathematics, University of Toronto,

Toronto, ON, M5S 2E4, Canada

e-mail: gold@math.utoronto.ca

Mircea Voda, Department of Mathematics, University of Toronto,

Toronto, ON, M5S 2E4, Canada

e-mail: mvoda@math.utoronto.ca 